\title{
Non-Markovian entanglement dynamics of quantum continuous variable systems in thermal environments
}

\author{
Kuan-Liang Liu and Hsi-Sheng Goan* \\ Department of Physics and Center for Theoretical Sciences, National Taiwan University, Taipei, Taiwan \\ and National Center for Theoretical Sciences, Taipei 10617, Taiwan \\ (Received 2 February 2007; revised manuscript received 16 May 2007; published 14 August 2007)
}

\begin{abstract}
We study two continuous variable systems (or two harmonic oscillators) and investigate their entanglement evolution under the influence of non-Markovian thermal environments. The continuous variable systems could be two modes of electromagnetic fields or two nanomechanical oscillators in the quantum domain. We use the quantum open system method to derive the non-Markovian master equations of the reduced density matrix for two different but related models of the continuous variable systems. The two models both consist of two interacting harmonic oscillators. In model A, each of the two oscillators is coupled to its own independent thermal reservoir, while in model B the two oscillators are coupled to a common reservoir. To quantify the degrees of entanglement for bipartite continuous variable systems in Gaussian states, logarithmic negativity is used. We find that the dynamics of the quantum entanglement is sensitive to the initial states, the oscillatoroscillator interaction, the oscillator-environment interaction and the coupling to a common bath or to different, independent baths.
\end{abstract}

DOI: $10.1103 /$ PhysRevA.76.022312

PACS number(s): 03.67.Mn, 03.65.Ud, 03.65.Yz, 85.85.+j

\section{INTRODUCTION}

Quantum information and computation is a nascent and interdisciplinary field that exploits quantum effects to compute and process information in ways that are faster or more efficient than, or even impossible, on conventional computers or information processing devices. This field has typically concerned itself with the manipulations of discrete systems such as quantum bits (qubits). Recently, the extension to continuous variables such as position, momentum, or the quadrature amplitudes of electromagnetic fields has led to the illuminating concept of continuous variable quantuminformation processing [1]. This includes the experimental realization of quantum teleportation [2-4] and the demonstration of quantum key distribution $[5,6]$ for continuous optical fields, and the successful definition of the notion of universal quantum computation over continuous variables [1].

Advances in current technology have allowed the fabrication of very small mechanical cantilevers or oscillators with high frequencies, and allowed their operation and manipulation at very low temperatures $[7,8]$. In the regime when the individual mechanical vibration quanta are of the order of the thermal energy, the motion of the mechanical oscillators is close to or on the verge of the quantum limit. Recently investigations devoted to observing quantum effect in the truly solid-state mechanical oscillators have been reported [9-19]. The Hilbert space of quantized electromagnetic fields is equivalent to the Hilbert space of the quantum harmonic oscillators. Thus, in addition to quantum optics system, it may be possible to implement continuous variable quantuminformation processing in the nanomechanical oscillator systems. This is particularly interesting in that it provides a stepping stone toward quantum state control and a platform

\footnotetext{
*Electronic address: goan@phys.ntu.edu.tw
}

to explore the transition from the quantum to the classical world in mechanical systems that consist of many millions of atoms [14-19].

Quantum entanglement has been considered as a key resource in many aspects and applications of quantuminformation processing. In the real world, quantum coherence and entanglement of quantum systems will inevitably be influenced and degraded by the external environment. There are several investigations of decoherence and quantum entanglement of continuous variable systems under open system dynamics in the literature [20-31]. But in those investigations, the Markovian approximation or (and) the rotatingwave approximation (RWA) is (are) assumed. However, if a short time interval or regime, comparable with the environmental correlation time, is concerned, or if the environment is structured with a particular spectral density, then the nonMarkovian environmental effect could become significant. For example, in the case when high-speed quantuminformation processing is required, the non-Markovian effect becomes important since the typical characteristic time of the relevant system may be comparable with the reservoir correlation time. In additon, when the typical system characteristic time is comparable with the decoherence and dissipation times, the other approximation, the RWA, widely used in the quantum optics master equation [32] to describe open quantum systems, may not apply. This is particularly the case for nanomechanical oscillators (or beams) as their fundamental vibration frequency $\Omega$ can currently just reach a few gigahertz [33], still much smaller than the optical frequency of $10^{15} \mathrm{~Hz}$. Thus a detailed investigation of the non-Markovian quantum entanglement dynamics in a more general setting without the RWA is needed. The main purpose of this paper is to present such a detailed analysis. The analysis is, as mentioned, of great importance to quantum nanomechanical oscillator systems and its relevance to quantum optics systems is also obvious.

We study, in this paper, two harmonic oscillators in the quantum domain and investigate their entanglement evolu- 
Model A

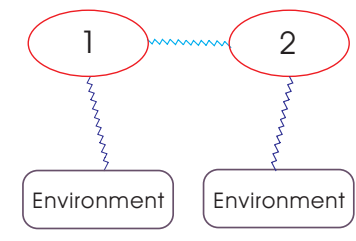

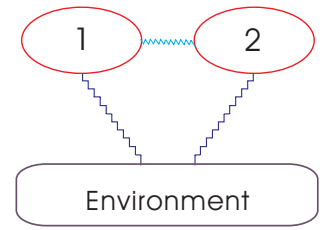

Model B
FIG. 1. (Color online) Schematic illustration of the two models invesigated. The two models bothe consist of two interacting harmonic oscillators. In model A, each of the two oscillators is coupled to its own independent thermal reservoir, while in model B the two oscillators are coupled to a common reservoir.

tion under the influence of thermal environments. [17-19,25,27,29] Two different but related models of harmonic oscillator systems are investigated (see Fig. 1). Model A consists of two interacting harmonic oscillators, each coupled to its own independent reservoir. The two oscillators may be envisaged to be sufficiently spatially far apart and may be relevant to the related setup for applications in quantum communication and teleportation. Model B also consists of two interacting harmonic oscillators, but both coupled to a common reservoir. They may be spatially close and could be useful for possible applications in quantum computation or other quantum-information processing tasks. The coupling between the two oscillators, and the coupling between the oscillators and the environments, are, if present, all bilinear in their respective positions (or coordinates). In practice, there may not be direct interaction between two optical fields, and this can be simply achieved by setting the coupling between the two oscillators to be zero in our models. On the other hand, a controllable and tunable interaction between two nanomechanical oscillators can be introduced by applying a voltage made from a metallic film fabricated on their surfaces $[10,19,34]$. Thus the two models discussed here are applicable to quantum nanomechanical oscillator systems and quantum optics systems.

In the context of two modes of an electromagnetic field embedded in a thermal environment, Ref. [25] derived a condition which states that if the state of the two modes is initially sufficiently squeezed, it will always remain entangled independently of the strength of the coupling to the environment. The model studied in Ref. [25] is the same as our model $\mathrm{B}$ but without the interaction between the two oscillators. The conclusion in Ref. [25] was derived however using the RWA and Markovian master equation. Here we investigate whether the condition presented in Ref. [25] is still valid or needs some modification in the non-Markovian case. We find that in the case of non-Markovian dynamics the condition depends also on the interaction strength between the system and environment.

This paper is organized as follows. In Sec. II the Hamiltonian and non-Markovian master equations for our two models are presented. In Sec. III we introduce the concepts of renormalization and the counterterm. In Sec. IV we introduce the logarithmic negativity to quantify entanglement for the two oscillators in our models. This entanglement monotone of logarithmic negativity is conveniently computable for general Gaussian states, and could provide a proper quantification of entanglement particularly for two-mode Gaussian states. We thus discuss the covariance matrix and two-mode squeezed vacuum state, a subclass of Gaussian states which we will use as initial states. In Sec. V, the covariance matrix evolution equations obtained from the master equations or the Fokker-Planck equations of Wigner function are described. In Sec. VI we present and discuss our results of entanglement dynamics based on the logarithmic negativity calculated through the evolution equations of the covariance matrix. Finally, we investigate whether the entanglement survival (or separability time) condition under the RWAMarkovian approximation in Ref. [25] is still valid in the non-Markovian case. A conclusion is given in Sec. VII.

\section{HAMILTONIAN AND NON-MARKOVIAN MASTER EQUATIONS OF TWO MODELS}

In this section we introduce two different but related models considered in this paper (see Fig. 1) and derive their corresponding quantum master equations up to the second order with respect to the system-environment coupling constant. The two models both consist of two interacting harmonic oscillators. In model A, each of the two oscillators is coupled to its own independent thermal reservoir, while the two oscillators are coupled to a common thermal reservoir in model B.

The Hamiltonian of the system of interest for the two models can be written as

$$
H_{s}=H_{s 1}+H_{s 2}+V_{12}
$$

where $H_{s 1}$ and $H_{s 2}$ are the Hamiltonian of the two subsystems, respectively, and $V_{12}$ is the interaction between them. They can be written as

$$
\begin{gathered}
H_{s 1}=\frac{p_{x}^{2}}{2 M_{x}}+\frac{1}{2} M_{x} \Omega_{x}^{2} x^{2}, \\
H_{s 2}=\frac{p_{y}^{2}}{2 M_{y}}+\frac{1}{2} M_{y} \Omega_{y}^{2} y^{2}, \\
V_{12}=\lambda x y,
\end{gathered}
$$

where $M_{x}$ and $M_{y}$ are the masses and $\Omega_{x}$ and $\Omega_{y}$ are the frequencies of the two subsystems (oscillators), respectively, and $\lambda$ is the coupling constant between the two subsystems. To consider the case of two noninteracting oscillators, we can simply set $\lambda$ to zero.

We assume that the environments can be described as ensembles of harmonic oscillators and interact bilinearly through their position operators with the system. The Hamiltonian of the two independent environments for model A is thus 


$$
\begin{aligned}
H_{\varepsilon}= & H_{\varepsilon 1}+H_{\varepsilon 2}=\sum_{n}\left(\frac{p_{n}^{(1)^{2}}}{2 m_{n}^{(1)}}+\frac{1}{2} m_{n}^{(1)} \omega_{n}^{(1)^{2}} q_{n}^{(1)^{2}}\right) \\
& +\sum_{n}\left(\frac{p_{n}^{(2)^{2}}}{2 m_{n}^{(2)}}+\frac{1}{2} m_{n}^{(2)} \omega_{n}^{(2)^{2}} q_{n}^{(2)^{2}}\right)
\end{aligned}
$$

and the interaction between the two subsystems and reservoirs for model A is

$$
V=V_{1}+V_{2}=\sum_{n} \lambda_{n}^{(1)} q_{n}^{(1)} x+\sum_{n} \lambda_{n}^{(2)} q_{n}^{(2)} y,
$$

where $\lambda_{n}^{(1)}$ and $\lambda_{n}^{(2)}$ are the coupling strengths to their own individual reservoirs, respectively.

On the other hand, the Hamiltonian of the environment for the two subsystems coupled to a common reservoir (model B) is

$$
H_{\varepsilon}=\sum_{n}\left(\frac{p_{n}^{2}}{2 m_{n}}+\frac{1}{2} m_{n} \omega_{n}^{2} q_{n}^{2}\right),
$$

and the coupling between two subsystems and reservoirs for model $\mathrm{B}$ are

$$
V=V_{1}+V_{2}=\sum_{n} \lambda_{n}^{(1)} q_{n} x+\sum_{n} \lambda_{n}^{(2)} q_{n} y .
$$

Using the perturbative expansion to the second order in the system-environment coupling strength, we obtain the equation of motion for the reduced density matrix $\rho(t)$ of the system of interest as [35]

$$
\begin{aligned}
\dot{\rho}(t)= & \frac{1}{i \hbar}\left[H_{s}, \rho(t)\right]-e^{-(i / \hbar) H_{s} t} \\
& \times\left(\frac{1}{\hbar^{2}} \int_{0}^{t} d t_{1} \operatorname{Tr}_{\varepsilon}\left[\tilde{V}(t),\left[\tilde{V}\left(t_{1}\right), \widetilde{\rho}(t) \otimes \rho_{\varepsilon}\right]\right]\right) e^{(i / \hbar) H_{s} t},
\end{aligned}
$$

where $\tilde{\rho}$ and $\tilde{V}$ are the density matrix of the system and the interaction between the system and environment in the interaction picture, respectively, and $\operatorname{Tr}_{\varepsilon}$ indicates tracing over environment degrees of freedom with respect to the thermal environment density matrix $\rho_{\varepsilon}$. In obtaining Eq. (9), we have also used the fact that the system-environment interaction is bilinear in their respective positions (or displacements) so that the first-order term $\operatorname{Tr}_{\varepsilon}\left[\tilde{V}(t) \rho_{\varepsilon}\right]=0$. We will derive the non-Markovian master equations for our models using Eq. (9) without making any further approximation. For simplicity, we assume the masses, resonance frequencies, and coupling strengths to the environments are the same for the two oscillators. That is, $M_{x}=M_{y}=M, \Omega_{x}=\Omega_{y}=\Omega$, and $\lambda_{n}^{(1)}=\lambda_{n}^{(2)}$ $=\lambda_{n}$. We present the derived non-Markovian master equations below.

\section{A. Model A}

The master equation for two coupled oscillators, each coupled to its own reservoir, of model A can be obtained as

$$
\begin{aligned}
\dot{\rho}(t)= & \frac{1}{i \hbar}\left[H_{s}+\frac{1}{4} M \widetilde{\Omega}_{1}^{2}(t)(x-y)^{2}+\frac{1}{4} M \widetilde{\Omega}_{2}^{2}(t)(x+y)^{2}, \rho\right] \\
& -\frac{i}{2 \hbar} \gamma_{1}(t)\left[x-y,\left\{p_{x}-p_{y}, \rho\right\}\right] \\
& -\frac{i}{2 \hbar} \gamma_{2}(t)\left[x+y,\left\{p_{x}+p_{y}, \rho\right\}\right]-\frac{1}{2} D_{1}(t)[x-y,[x-y, \rho]] \\
& +\frac{1}{2} D_{2}(t)[x+y,[x+y, \rho]]-\frac{1}{2 \hbar} f_{1}(t)\left[x-y,\left[p_{x}-p_{y}, \rho\right]\right] \\
& +\frac{1}{2 \hbar} f_{2}(t)\left[x+y,\left[p_{x}+p_{y}, \rho\right]\right] .
\end{aligned}
$$

Here the time-dependent coefficient $\widetilde{\Omega}_{i}^{2}(t)$ is called the frequency shift, $\gamma_{i}(t)$ is the dissipation coefficient, and $D_{i}(t)$ and $f_{i}(t)$ represent the diffusion coefficients. They can be written as

$$
\begin{gathered}
\widetilde{\Omega}_{i}^{2}(t)=-\frac{2}{M} \int_{0}^{t} d t^{\prime} \cos \left(\Omega_{i} t^{\prime}\right) \eta\left(t^{\prime}\right), \\
\gamma_{i}(t)=\frac{1}{M \Omega_{i}} \int_{0}^{t} d t^{\prime} \sin \left(\Omega_{i} t^{\prime}\right) \eta\left(t^{\prime}\right), \\
D_{i}(t)=\frac{1}{\hbar} \int_{0}^{t} d t^{\prime} \cos \left(\Omega_{i} t^{\prime}\right) \nu\left(t^{\prime}\right), \\
f_{i}(t)=-\frac{1}{M \Omega_{i}} \int_{0}^{t} d t^{\prime} \sin \left(\Omega_{i} t^{\prime}\right) \nu\left(t^{\prime}\right),
\end{gathered}
$$

where $i=1,2$, and the frequencies $\Omega_{1}$ and $\Omega_{2}$ due to the interaction $\lambda$ between the two oscillators are

$$
\begin{aligned}
& \Omega_{1}=\sqrt{\Omega^{2}-\lambda / M}, \\
& \Omega_{2}=\sqrt{\Omega^{2}+\lambda / M} .
\end{aligned}
$$

The two kernels $\eta\left(t^{\prime}\right)$ and $\nu\left(t^{\prime}\right)$ appearing in Eqs. (11)-(14) are, respectively, the so-called dissipation and noise kernels and are defined as

$$
\begin{gathered}
\eta(t)=\frac{1}{2 \hbar} \sum_{n} \lambda_{n}^{2}\left\langle\left[q_{n}(t), q_{n}(0)\right]\right\rangle=\int_{0}^{\infty} d \omega J(\omega) \sin (\omega t), \\
\nu(t)=\frac{1}{2 \hbar} \sum_{n} \lambda_{n}^{2}\left\langle\left\{q_{n}(t), q_{n}(0)\right\}\right\rangle \\
=\int_{0}^{\infty} d \omega J(\omega) \cos (\omega t)[1+2 N(\omega)],
\end{gathered}
$$

where 


$$
N(\omega)=\frac{1}{e^{\hbar \omega / k_{B} T}-1}
$$

is the mean occupation number of the environmental oscillators, and

$$
J(\omega)=\sum_{n} \frac{\lambda_{n}^{2}}{2 m_{n} \omega_{n}} \delta\left(\omega-\omega_{n}\right)
$$

is the spectral density of the environments. Note that the environment position operator $q_{n}(t)$ in Eqs. (17) and (18) should be $q_{n}^{(i)}(t)$ for different environments $i$. We could in principle deal with this situation, but for simplicity we assume that they have the same corresponding correlations even though the two environments are independent of each other. It is also worth noting that the frequency shift (11) and dissipation coefficient (12) depend only on the dissipation kernel (17), while the diffusion coefficients (13) and (14) in the approximation of the perturbative expansion depend only on the noise kernel (18), and are thus temperature dependent. We can see from Eq. (10) that the term proportion to $\gamma_{i}(t)$ is responsible for relaxation and the term proportional to $D_{i}(t)$ is the main cause of decoherence. The spectral density specifies the structure and properties of the environment and thus determines the environmental influence on the dynamics of the system of interest. In fact, the time evolution behavior of the coefficients of the quantum master equation is rather different for environments with different spectral content.

In principle, we could deal with any given form of the spectral density. But as a particular example, we use the following form of spectral density to specify the environments $[36,37]$ :

$$
J(\omega)=\frac{2}{\pi} M \gamma_{0} \omega\left(\frac{\omega}{\Lambda}\right)^{n-1} e^{-\omega^{2} / \Lambda^{2}},
$$

where $\Lambda$ is the cutoff frequency, $\gamma_{0}$ is a constant characterizing the strength of the interaction with the environment, and $M$ is the system mass. The environment is said to be Ohmic if in the physical range of frequencies $(\omega<\Lambda)$ the spectral density is proportional to $\omega$. And it is said to be supra-Ohmic if $J(\omega)$ is proportional to $\omega^{n}, n>1$, or subOhmic if $n<1$. For simplicity, in the following we focus on Ohmic baths, i.e., the case of $n=1$ in Eq. (21).

If we let $\lambda=0$, then this model reduces to two noninteracting oscillators, each coupled to its own reservoir. The master equation (10) in this instance also reduces to the case of just putting two sets of the quantum Brownian motion master equations [35] together:

$$
\begin{aligned}
\dot{\rho}(t)= & \frac{1}{i \hbar}\left[H_{s}+\frac{1}{2} M \widetilde{\Omega}^{2}(t)\left(x^{2}+y^{2}\right), \rho\right]-\frac{i}{\hbar} \gamma(t)\left(\left[x,\left\{p_{x}, \rho\right\}\right]\right. \\
& \left.+\left[y,\left\{p_{y}, \rho\right\}\right]\right)-D(t)([x,[x, \rho]]+[y,[y, \rho]]) \\
& -\frac{1}{\hbar} f(t)\left(\left[x,\left[p_{x}, \rho\right]\right]+\left[y,\left[p_{y}, \rho\right]\right]\right)
\end{aligned}
$$

where the time-dependent coefficients $\widetilde{\Omega}^{2}(t), \gamma(t), D(t)$, and $f(t)$ are defined correspondingly to those in Eqs. (11)-(14) with $\lambda=0$.

\section{B. Model B}

The master equation for two interacting oscillators, coupled to a common reservoir, of model B can be derived and written as

$$
\begin{aligned}
\dot{\rho}(t)= & \frac{1}{i \hbar}\left[H_{s}+\frac{1}{2} M \widetilde{\Omega}_{2}^{2}(t)(x+y)^{2}, \rho\right] \\
& -\frac{i}{\hbar} \gamma_{2}(t)\left[x+y,\left\{p_{x}+p_{y}, \rho\right\}\right]-D_{2}(t)[x+y,[x+y, \rho]] \\
& -\frac{1}{\hbar} f_{2}(t)\left[x+y,\left[p_{x}+p_{y}, \rho\right]\right] .
\end{aligned}
$$

The time-dependent coefficients are the same as those in Eqs. (11)-(14) with $i=2$ in model A. Note that, compared with Eq. (10), the mode $(x-y)$ is absent in Eq. (23). This is a consequence of both the assumptions of $M_{x}=M_{y}=M, \Omega_{x}$ $=\Omega_{y}=\Omega$, and $\lambda_{n}^{(1)}=\lambda_{n}^{(2)}=\lambda_{n}$ which we make to simplify the calculation, as well as the nature of model B which is coupled to a common bath with the variable $(x+y)$. This can also be inferred from the effective factor of 2 difference in the corresponding coefficients of terms containing the $(x$ $+y$ ) variable between Eqs. (10) and (23). In the derivation of the master equation in model $\mathrm{B}$, some type of addition makes the coefficients of the $(x+y)$ mode twice larger and some type of cancellation makes the $(x-y)$ mode absent in Eq. (23). We note that, despite being derived perturbatively, the master equations for the two models seem to be very similar to their exact counterparts, which are also time convolutionless with time-dependent coefficients [37,38].

\section{RENORMALIZATION AND TIME-DEPENDENT COEFFICIENTS}

We note that, due to the interaction with the environment, the frequency shift term $\widetilde{\Omega}_{i}^{2}(t)$ of Eq. (11) diverges as the cutoff frequency $\Lambda \rightarrow \infty$ and thus is not physical. Thus a regularization procedure is needed for the frequency renormalization. There are two different views on this renormalization $[39,40]$. One view is that, starting from the original Hamiltonian, the frequency can be made finite, by a renormalization of frequency, from its bare to its physical value. Thus by combining that which involves the frequency in the master equation, the physical frequency, which is the quantity that can be measured in the laboratory, is defined as

$$
\Omega_{p}^{2}(t)=\Omega^{2}+\widetilde{\Omega}^{2}(t),
$$

where $\Omega$ is the bare frequency in the Hamiltonian. In this case, the physical frequency is taken to be finite and the bare frequency is taken to be infinite as $\Lambda \rightarrow \infty$ in order to cancel the divergent contribution from $\widetilde{\Omega}^{2}(t)$. Thus the bare frequency has no direct physical significance. Although it may not be exactly the same, this view of renormalization has an analogy in solid state physics where, for example, electrons are attributed an effective mass to take into account their interaction with the lattice or/and other electrons.

An alternative view of renormalization is to regard the frequency $\Omega$ in the original Hamiltonian as a finite renormal- 
ized frequency $\Omega_{r}$. The fact that this Hamiltonian does not give finite frequency then requires that extra terms be added to the Hamiltonian to cancel the divergence. These terms are called counterterms [39-42]. This view of renormalization is in fact more commonly adopted in high-energy physics or quantum field theory. In the context of a quantum Brownian motion model, the total Hamiltonian in this case can be written as

$$
\begin{aligned}
H= & \frac{p^{2}}{2 M}+\frac{1}{2} M \Omega_{r}^{2} x^{2}+\sum_{n}\left(\frac{p_{n}^{2}}{2 m_{n}}+\frac{1}{2} m_{n} \omega_{n}^{2} q_{n}^{2}+\lambda_{n} q_{n} x\right) \\
& +\sum_{n} \frac{1}{2} \frac{\lambda_{n}^{2}}{m_{n} \omega_{n}^{2}} x^{2} \\
= & \frac{p^{2}}{2 M}+\frac{1}{2} M \Omega_{r}^{2} x^{2}+\sum_{n}\left[\frac{p_{n}^{2}}{2 m_{n}}+\frac{1}{2} m_{n} \omega_{n}^{2}\left(q_{n}+\frac{\lambda_{n}}{m_{n} \omega_{n}^{2}} x\right)^{2}\right] .
\end{aligned}
$$

The last term in Eq. (25) can be viewed as a frequency counterterm $[41,42]$ with a frequency defined as

$$
\Omega_{c}^{2}=\frac{1}{M} \sum_{n} \frac{\lambda_{n}^{2}}{m_{n} \omega_{n}^{2}}=2 \int_{0}^{\infty} d \omega \frac{J(\omega)}{\omega} .
$$

We can see that at large times the frequency shift $\widetilde{\Omega}^{2}(t)$ is negative and equals $-\Omega_{c}^{2}$. The added counterterm is to cancel the frequency shift at long times and ensure that the system cannot lower its potential energy below the original (renormalized) value. The physical frequency in this case is $\Omega_{p}^{2}$ $=\Omega_{r}^{2}+\Omega_{c}^{2}+\widetilde{\Omega}^{2}(t)$ and equals $\Omega_{r}^{2}$ at long times. We will take this view of renormalization, so the original frequency $\Omega$ in the time-dependent coefficients of the master equations, Eqs. (10) and (23), should be replaced by $\Omega \rightarrow \Omega_{r}$.

\section{LOGARITHMIC NEGATIVITY AND TWO-MODE GAUSSIAN STATES}

The purpose of this paper is to focus on the entanglement dynamics for the reduced density matrix of the two oscillators in our models. The derived master equations are generally partial differential equations with time-dependent coefficients. Consequently, computing the time evolution solution for the density matrix operator explicitly and then using it to calculate directly the dynamics of entanglement is still considered difficult. But the problem becomes much more tractable if we restrict the states to be Gaussian states. Since the couplings in our models are all bilinear in their respective positions (displacements) and the effects of the environments in the master equations have operator structure no more than quadratic in the momenta or/and positions of the two oscillators, an initial Gaussian state would remain Gaussian in its subsequent time evolution. So, for simplicity, we will consider in the following Gaussian initial states for the two oscillators. Any Gaussian state can be completely characterized by its corresponding covariance matrix. We will see below that the time evolution of the covariance matrix is easier to calculate than that of the density matrix.
A set of Gaussian states is the set of states with Gaussian characteristic functions and quasiprobability distributions of the Wigner function [43]. The Wigner quasiprobability distribution function is defined in terms of density matrix $\rho(t)$ as

$$
\begin{aligned}
W(\boldsymbol{q}, \boldsymbol{p}, t) \equiv & \left(\frac{1}{2 \pi \hbar}\right)^{n} \int_{-\infty}^{\infty} d \boldsymbol{\xi}\left\langle\boldsymbol{q}-\frac{\boldsymbol{\xi}}{2}|\rho(t)| \boldsymbol{q}\right. \\
& \left.+\frac{\boldsymbol{\xi}}{2}\right\rangle \exp \left(\frac{i \boldsymbol{p} \cdot \boldsymbol{\xi}}{\hbar}\right) .
\end{aligned}
$$

So a zero-mean Gaussian state is described, for example, by the Wigner function as

$$
W(\mathbf{X})=\frac{1}{4 \pi^{n} \sqrt{\operatorname{det} \mathbf{V}}} \exp \left(-\frac{1}{2} \mathbf{X} \mathbf{V}^{-1} \mathbf{X}^{T}\right)
$$

where $\mathbf{V}$ is the covariance matrix and $\mathbf{X}$ represents the vector $\left(x_{1}, p_{1}, x_{2}, p_{2}, \ldots, x_{n}, p_{n}\right)$. The zero mean denotes $\left\langle X_{i}\right\rangle=0$, and this can be changed at will using local unitary displacement operators. So we can set $\left\langle X_{i}\right\rangle=0$ without loss of generality.

The matrix elements of the covariance matrix $\mathbf{V}$ are defined as

$$
\begin{aligned}
V_{i, j} & \equiv\left\langle\left\{\Delta \hat{X}_{i}, \Delta \hat{X}_{j}\right\}\right\rangle=\operatorname{Tr}\left(\left\{\Delta \hat{X}_{i}, \Delta \hat{X}_{j}\right\} \hat{\rho}\right) \\
& =\int d^{4} X \Delta X_{i} \Delta X_{j} W(X),
\end{aligned}
$$

where $\left\{\Delta \hat{X}_{i}, \Delta \hat{X}_{j}\right\}=\left(\Delta \hat{X}_{i} \Delta \hat{X}_{j}+\Delta \hat{X}_{j} \Delta \hat{X}_{i}\right) / 2, \Delta \hat{X}_{i}=\hat{X}_{i}-\left\langle\hat{X}_{i}\right\rangle$, and $\Delta X_{i}=X_{i}-\left\langle X_{i}\right\rangle$. The average of the operator $\hat{X}_{i},\left\langle\hat{X}_{i}\right\rangle$, means $\operatorname{Tr}\left(\hat{X}_{i} \hat{\rho}\right)$, and $\left\langle X_{i}\right\rangle$ denotes an average of a variable $X_{i}$ with respect to the Wigner function distribution $W(\mathbf{X})$, so $\left\langle\hat{X}_{i}\right\rangle$ equals $\left\langle X_{i}\right\rangle$. With this definition, we could transfer the problem of solving a time-dependent partial differential equation of the density matrix into a problem of solving first-order-intime, coupled linear ordinary differential equations of the covariance matrix elements. This could be done by first transferring the master equations to the Fokker-Planck equation for the Wigner function, and then finding the coupled differential evolution equation for the covariance matrix elements using Eq. (30) and the Fokker-Planck equation (see Sec. V).

We will use the logarithmic negativity to quantify the degrees of entanglement of the infinite-dimensional bipartite system states of the two oscillators. The logarithmic negativity of a bipartite system consisting of two subsystems A and $\mathrm{B}$ is [44]

$$
E_{N}(\rho) \equiv \log _{2}\left\|\rho^{T_{B}}\right\|_{1},
$$

where $\rho^{T_{B}}$ means the partial transpose of a (mixed) state density matrix operator $\rho$ with respect to subsystem $B$. That is to say, $\left\langle i_{A}, j_{B}\left|\rho^{T_{B}}\right| k_{A}, l_{B}\right\rangle \equiv\left\langle i_{A}, l_{B}|\rho| k_{A}, j_{B}\right\rangle$ for any arbitrary orthonormal product basis which belongs to the tensor product of the Hilbert space of the combinative system $A$ and $B$. The operation $\|\cdot\|_{1}$ denotes the trace norm, and the trace norm of any Hermitian operator $H$ is defined as $\|H\|_{1}$ $\equiv \operatorname{Tr}|H| \equiv \operatorname{Tr} \sqrt{H^{\dagger} H}$. 
Despite not being convex, the logarithmic negativity is a full entanglement monotone under local operations and classical communication [45] and constitutes an upper bound to the distillable entanglement [44]. For the particular case of two-mode Gaussian states, the logarithmic negativity could actually provide an appropriate quantification of quantum entanglement. Vidal and Werner [44] demonstrated that logarithmic negativity is computable for general Gaussian states. For two-mode Gaussian states, it can be furthermore shown that the logarithmic negativity can be represented as [43]

$$
E_{N}(\rho)=\max \left(0,-\log _{2} 2 V_{s}\right),
$$

where $V_{s}$ is the smallest sympletic eigenvalue of the partially transposed covariance matrix of the two-mode Gaussian states. Equation (32) is a simple decreasing function of $V_{s}$, which quantifies the degree of violation of the necessary and sufficient separability criterion of the positivity of partial transpose $[47,43]$. For $V_{s} \geqslant 1 / 2$ the state is separable; otherwise it is entangled. So the smallest partially transposed sympletic eigenvalue $V_{s}$ alone completely qualifies and quantifies the quantum entanglement of a two-mode Gaussian state [43]. That is, the smaller the value of $V_{s}$, the more entangled the corresponding two-mode Gaussian state. As a result, the logarithmic negativity may be regarded as a suitable entanglement quantification indicator for two-mode Gaussian states.

The partially transposed sympletic eigenvalues $V_{i}$ are the symplectic eigenvalues of $\mathbf{V}^{T_{B}}$, and $\mathbf{V}^{T_{B}}$ can be written down in a compact form [18] as

$$
\mathbf{V}^{T_{B}}=P \mathbf{V} P,
$$

where

$$
P=\left(\begin{array}{ll}
1 & 0 \\
0 & 1
\end{array}\right) \oplus\left(\begin{array}{cc}
1 & 0 \\
0 & -1
\end{array}\right)
$$

and $A \oplus B$ means the block-diagonal matrix with the matrices $A$ and $B$ as diagonal entries. The symplectic eigenvalues are the positive square roots of the standard eigenvalues of $-\sigma \mathbf{V}^{T_{B}} \sigma \mathbf{V}^{T_{B}}$ or the absolute value of the eigenvalues of $i \sigma \mathbf{V}^{T_{B}}$. Here $\sigma$ is called the symplectic matrix from the commutation relations $\left[\hat{x}_{i}, \hat{x}_{j}\right]=i \hbar \sigma_{i, j}$ which is given by

$$
\sigma=\left(\begin{array}{ll}
J & 0 \\
0 & J
\end{array}\right) \text { and } J=\left(\begin{array}{cc}
0 & 1 \\
-1 & 0
\end{array}\right) .
$$

The logarithmic negativity in the form of Eq. (32) is much easier to compute than that defined in Eq. (31).

One subclass of two-mode Gaussian states is the so-called two-mode squeezed vacuum states. The position and momentum wave functions for the two-mode squeezed vacuum state with a squeezing parameter $r$ are [1]

$$
\psi(x, y)=\sqrt{\frac{2}{\pi}} \exp \left[-e^{-2 r}(x+y)^{2} / 2-e^{2 r}(x-y)^{2} / 2\right],
$$

$$
\bar{\psi}\left(p_{x}, p_{y}\right)=\sqrt{\frac{2}{\pi}} \exp \left[-e^{-2 r}\left(p_{x}-p_{y}\right)^{2} / 2-e^{2 r}\left(p_{x}+p_{y}\right)^{2} / 2\right] .
$$

They approach $C \delta(x-y)$ and $C \delta\left(p_{x}+p_{y}\right)$, respectively, in the limit of infinite squeezing $r \rightarrow \infty$, where $C$ is some constant. The corresponding Wigner function of the two-mode squeezed vacuum state is then [1]

$$
\begin{aligned}
W(\mathbf{X})= & \frac{4}{\pi^{2}} \exp \left\{-e^{-2 r}\left[(x+y)^{2}+\left(p_{x}-p_{y}\right)^{2}\right]-e^{2 r}(x-y)^{2}\right. \\
& \left.\left.+\left(p_{x}+p_{y}\right)^{2}\right]\right\} .
\end{aligned}
$$

In the limit of infinite squeezing $r \rightarrow \infty$, this Wigner function approaches $C \delta(x-y) \delta\left(p_{x}+p_{y}\right)$, corresponding to the original (perfectly correlated and maximally entangled) EinsteinPodolsky-Rosen state, while at $r=0$, two-mode state corresponds to a separable (disentangled) state. Two-mode squeezed vacuum states are routinely generated in quantum optics laboratories and have been used in most implementations of continuous variable quantum-information protocols $[1,2,46]$. It has also been proposed recently that a two-mode squeezed state could be generated for two nanomechanical oscillators that act as the two opposite sections, suspended from the substrate, of a dc-superconducting quantum interference device (SQUID) loop [16].

The two-mode squeezed vacuum states, from Eqs. (29) and (38), can be completely characterized by the following covariance matrix $[47,48]$ :

$$
\mathbf{V} \equiv\left(\begin{array}{cccc}
a & 0 & -c & 0 \\
0 & a & 0 & c \\
-c & 0 & a & 0 \\
0 & c & 0 & a
\end{array}\right),
$$

where $a$ and $c$ are

$$
a=\cosh (2 r) / 2, \quad c=\sinh (2 r) / 2 .
$$

For simplicity, we will use the two-mode squeezed vacuum states as the initial states of the two quantum oscillators in our models throughout the paper.

\section{EVOLUTION EQUATIONS OF THE COVARIANCE MATRIX ELEMENTS}

By the definition of the Wigner function in Eq. (28), the corresponding function for $\dot{\rho}(t)$ is

$$
\dot{W}(\boldsymbol{q}, \boldsymbol{p}, t)=\left(\frac{1}{2 \pi \hbar}\right)^{n} \int_{-\infty}^{\infty} d \boldsymbol{\xi}\left\langle\boldsymbol{q}-\frac{\boldsymbol{\xi}}{2}|\dot{\rho}(t)| \boldsymbol{q}+\frac{\boldsymbol{\xi}}{2}\right\rangle \exp \left(\frac{i \boldsymbol{p} \cdot \boldsymbol{\xi}}{\hbar}\right) .
$$

Using Eqs. (28) and (41), and after straightforward but somewhat tedious calculations, we can obtain the Fokker-Planck equations of the Wigner function corresponding to the master equations (10) and (23). From the Fokker-Planck equation of the Wigner function and Eq. (30), we can obtain coupled first-order ordinary differential equations with time- 
dependent coefficients for all elements of the covariance matrix. Due to the symmetrical property of the covariance matrix, i.e., $V_{12}=V_{21}, V_{24}=V_{42}$, etc., we need only ten essential components of the covariance matrix in the bipartite system here instead of 16 components. For example, we obtain for model B with the renormalized frequency $\Omega \rightarrow \Omega_{r}$ :

$\dot{V}_{11}=2 V_{12}$

$$
\begin{aligned}
\dot{V}_{12}= & -\left[\Omega_{r}^{2}+\Omega_{c}^{2}+\widetilde{\Omega}_{2}^{2}(t)\right] V_{11}-2 \gamma_{2}(t) V_{12} \\
& -\left[\Omega_{c}^{2}+\widetilde{\Omega}_{2}^{2}(t)+\lambda / M\right] V_{13}-2 \gamma_{2}(t) V_{14}+V_{22}-\hbar f_{2}(t),
\end{aligned}
$$

$\dot{V}_{13}=V_{14}+V_{23}$,

$$
\begin{aligned}
\dot{V}_{14}= & -\left[\Omega_{c}^{2}+\widetilde{\Omega}_{2}^{2}(t)+\lambda / M\right] V_{11}-2 \gamma_{2}(t) V_{12} \\
& -\left[\Omega_{r}^{2}+\Omega_{c}^{2}+\widetilde{\Omega}_{2}^{2}(t)\right] V_{13}-2 \gamma_{2}(t) V_{14}+V_{24}-\hbar f_{2}(t),
\end{aligned}
$$$$
\dot{V}_{22}=-2\left[\Omega_{r}^{2}+\Omega_{c}^{2}+\widetilde{\Omega}_{2}^{2}(t)\right] V_{12}-4 \gamma_{2}(t) V_{22}
$$$$
-2\left[\Omega_{c}^{2}+\widetilde{\Omega}_{2}^{2}(t)+\lambda / M\right] V_{23}-4 \gamma_{2}(t) V_{24}+2 \hbar^{2} D_{2}(t),
$$$$
\dot{V}_{23}=-\left[\Omega_{r}^{2}+\Omega_{c}^{2}+\widetilde{\Omega}_{2}^{2}(t)\right] V_{13}-2 \gamma_{2}(t) V_{23}+V_{24}
$$$$
-\left(\Omega_{c}^{2}-\widetilde{\Omega}_{2}^{2}+\lambda\right) V_{33}-2 \gamma_{2} V_{34}-\hbar f_{2}(t),
$$

$$
\begin{aligned}
\dot{V}_{24}= & -\left[\Omega_{c}^{2}+\widetilde{\Omega}_{2}^{2}(t)+\lambda / M\right] V_{12}-\left[\Omega_{r}^{2}+\Omega_{c}^{2}+\widetilde{\Omega}_{2}^{2}(t)\right] V_{14} \\
& -2 \gamma_{2}(t) V_{22}-\left[\Omega_{r}^{2}+\Omega_{c}^{2}+\widetilde{\Omega}_{2}^{2}(t)\right] V_{23}-4 \gamma_{2}(t) V_{24}-\left[\Omega_{c}^{2}\right. \\
& \left.+\widetilde{\Omega}_{2}^{2}(t)+\lambda / M\right] V_{34}-2 \gamma_{2}(t) V_{44}+2 \hbar^{2} D_{2}(t),
\end{aligned}
$$

$\dot{V}_{33}=2 V_{34}$,

$$
\begin{aligned}
\dot{V}_{34}= & -\left[\Omega_{c}^{2}+\widetilde{\Omega}_{2}^{2}(t)\right] V_{13}-2 \gamma_{2}(t) V_{23}-\left[\Omega_{r}^{2}+\Omega_{c}^{2}+\widetilde{\Omega}_{2}^{2}(t)\right] V_{33} \\
& -2 \gamma_{2}(t) V_{34}+V_{44}-\hbar f_{2}(t),
\end{aligned}
$$$$
\dot{V}_{44}=-2\left[\Omega_{c}^{2}+\widetilde{\Omega}_{2}^{2}(t)+\lambda / M\right] V_{14}-4 \gamma_{2}(t) V_{24}
$$$$
-2\left[\Omega_{r}^{2}+\Omega_{c}^{2}+\widetilde{\Omega}_{2}^{2}(t)\right] V_{34}-4 \gamma_{2}(t) V_{44}+2 \hbar^{2} D_{2}(t) .
$$

Similar calculations can be performed for model A. The solutions of the coupled first-order ordinary differential equations in time are much easier to calculate than the partial differential equations of the Fokker-Planck equations or the quantum master equations. Solving for the time evolution of the covariance matrix elements, we can then obtain the entanglement dynamics through the computation of the logarithmic negativity using Eq. (32).

\section{RESULTS AND DISCUSSIONS}

We first report our numerical results of the nonMarkovian entanglement (logarithmic negativity) dynamics for our two models in two cases: (1) $\gamma_{0}=0$ (isolated) and (2)

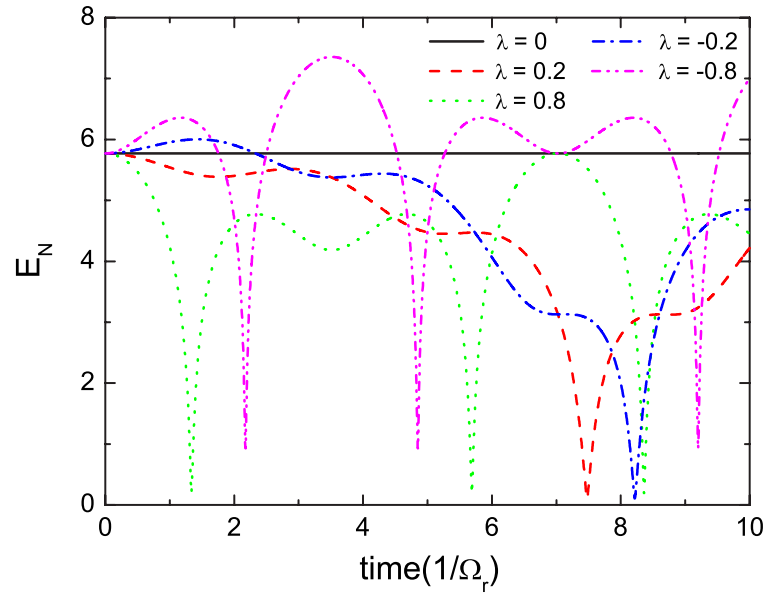

FIG. 2. (Color online) Time evolution of the logarithmic negativity of the two subsystems isolated from external environments $\left(\gamma_{0}=0\right)$ for the case of an initial two-mode squeezed vacuum state with a squeezing parameter $r=2$. The solid line stands for $\lambda=0$, dashed for $\lambda=0.2$, dotted for $\lambda=0.8$, dash-dotted for $\lambda=-0.2$, and dash-dot-dotted for $\lambda=-0.8$. In all the plots presented below, the parameters used are as follows. The environment temperature is at $k_{B} T=10 \hbar \Omega_{r}$, the cutoff frequency is $\Lambda=2000 \Omega_{r}$, and the interaction between the two subsystems $\lambda$ is in units of $M \Omega_{r}^{2}$.

$\gamma_{0}=6 \times 10^{-2} \Omega_{r}$, where $\gamma_{0}$ is a constant in the spectral density (21) and is related to the coupling strength to the environments, and $\Omega_{r}$ is the renormalized frequency of the subsystems. In all the plots presented below, the parameters used are as follows. The environment temperature is at $k_{B} T$ $=10 \hbar \Omega_{r}$, the cutoff frequency is $\Lambda=2000 \Omega_{r}$, and the interaction between two subsystems $\lambda$ is in units of $M \Omega_{r}^{2}$. Finally, we investigate whether the entanglement survival condition under the RWA-Markovian approximation in Ref. [25] is still valid in the non-Markovian case.

\section{A. Isolated system $\left(\gamma_{0}=0\right)$}

We plot the dynamics of the logarithmic negativity of the two models when they are isolated from the external envi-

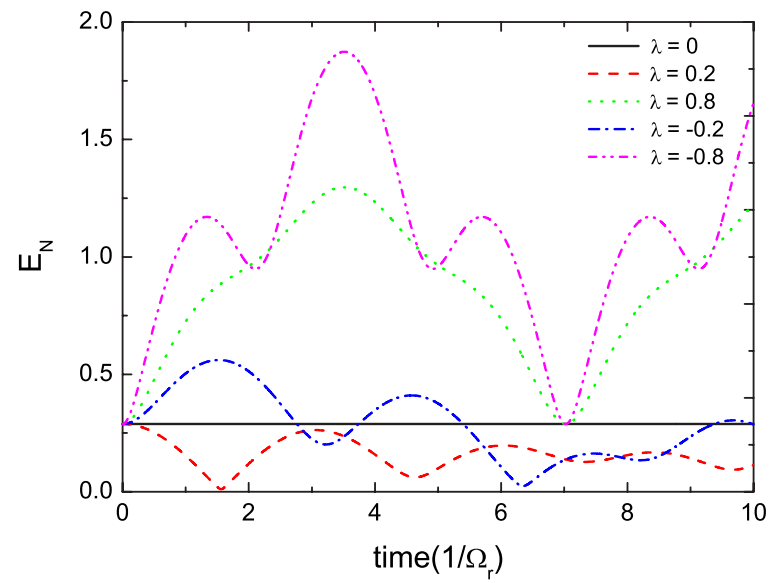

FIG. 3. (Color online) Time evolution of the logarithmic negativity for an initial two-mode squeezed vacuum state with a squeezing parameter $r=0.1$. Other conditions and plot caption are the same as in Fig. 2. 


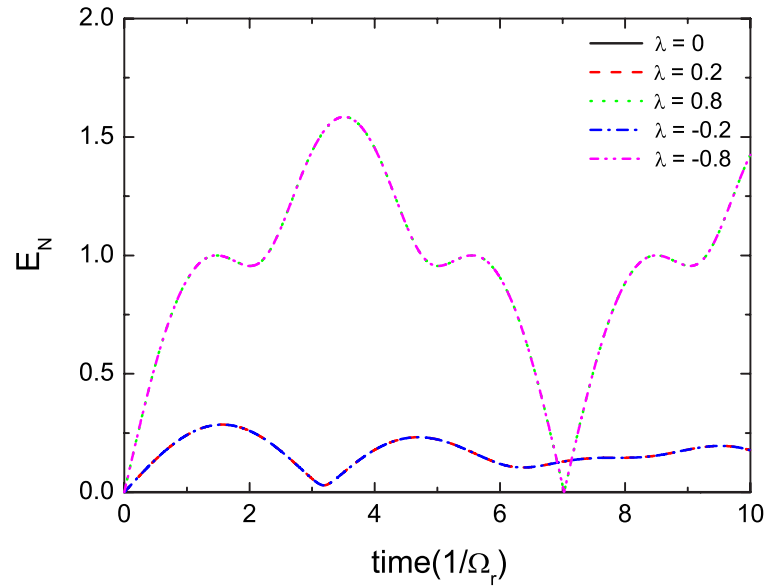

FIG. 4. (Color online) Time evolution of the logarithmic negativity for an initial two-mode squeezed vacuum state with a squeezing parameter $r=0$. Other conditions and plot caption are the same as in Fig. 2.

ronments in Figs. 2-4. Similar to that of an interacting discrete system of two qubits, the dynamics of entanglement of the two oscillators depends strongly on the initial states and on the interacting strength between them. When there is no interaction between the two subsystems $(\lambda=0)$ isolated from the environments, the time evolution of the logarithmic negativity maintains constant in Figs. 2-4, as it should. But the dynamics of the logarithmic negativity varies quasiperiodically for two interacting subsystems isolated from the external environments, and the smaller the value of the interaction strength $|\lambda|$ between the two oscillators, the longer is the quasiperiod of the logarithmic negativity. This can be seen from the plots of the entanglement dynamics on a longer time scale.

From Fig. 4, we see that the entanglement of the two subsystems can be generated from an initially separable state $(r=0)$ through their mutual interaction, and the larger the interaction strength, the larger the generation of the entanglement. We can also see from Fig. 4 that the entanglement dynamics for an initially separable state $(r=0)$ seems to be symmetric with respect to the change of $\lambda x y \leftrightarrow-\lambda x y$, while this is not the case for $r \neq 0$ (see Figs. 2 and 3). This may be due to the fact that for $r=0$ the initial wave function Eq. (36) or the Wigner function Eq. (38) is symmetric under the change of $x \leftrightarrow-x, y \leftrightarrow-y$, and $x y \leftrightarrow-x y$; while for $r \neq 0$, this symmetry is broken and the initial wave function Eq. (36) or the Wigner function Eq. (38) possesses the preferred entanglement in the relative position variable $(x-y)$ as compared to the variable $(x+y)$. So an attractive interaction $(\lambda$ $<0)$ seems to enhance this entanglement in $(x-y)$. This can be seen from Figs. 2-4; for a fixed value of the squeezing parameter $r$, if the interaction strength is attractive $(\lambda<0)$ then the entanglement grows initially with time. On the other hand, the entanglement decreases with time initially if the interaction strength is positive and smaller than a critical value, i.e., $0<\lambda<\lambda_{c}$. For example, in Fig. 3 the initial entanglement grows with time for $\lambda=0.8$, while it decreases with time for $\lambda=0.2$. Similarly, we may say that, for a fixed positive value of $\lambda>0$, there exists a critical initial squeez-

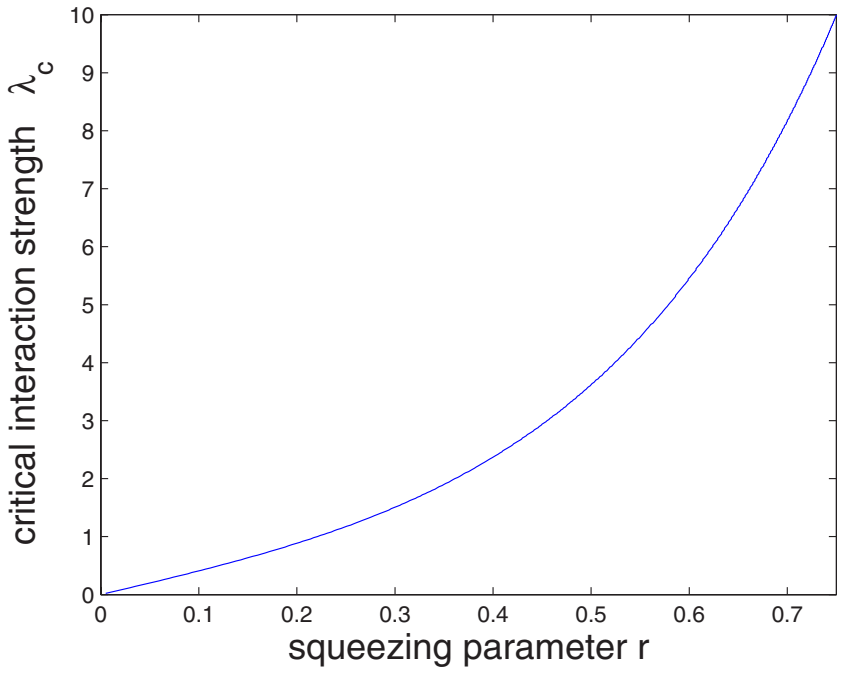

FIG. 5. (Color online) Positive interaction strength versus initial squeezing parameter phase diagram. The regime above (below) the critical line curve corresponds to the situation that the entanglement increases (decreases) with time initially.

ing parameter above which the entanglement initially decreases with time. Figure 5 shows the critical value line that separates these two situations in the phase diagram of the positive interaction strength $\lambda$ versus initial squeezing parameter $r$.

The general trend is that, when the entanglement grows with time initially, the entanglement is enhanced to reach maximum values at later times; while, if the entanglement decreases with time initially, the initial value of the entanglement is usually the maximum value. Thus, for two oscillators initially in a two-mode squeezed vacuum state with a squeezing parameter $r$, an attractive interaction $(\lambda<0)$ between the two oscillators is able to enhance their entanglement at later times. A repulsive interaction $(\lambda>0)$ can enhances the entanglement at later times if $\lambda>\lambda_{c}$, but the entanglement is no longer increased if $0<\lambda<\lambda_{c}$. For the same value of interaction strength between the two oscillators, the attractive interaction seems always to be better than the repulsive interaction as far as the maximum value of entanglement that can be reached at a later time is concerned.

\section{B. System coupled to environments $\left(\gamma_{0}=6 \times 10^{-2} \Omega_{r}\right)$}

In Figs. 6 and 7, we plot the dynamics of the logarithmic negativity of the two subsystems coupled to the environments for our two models with different initial states of $r$ $=2$ and 0 , respectively. Compared with the corresponding $\gamma_{0}=0$ cases in Fig. 2, the oscillatory phenomena due to the influence of the environments disappear except in Fig. 6(b). It can also be seen from Fig. 6(a) that the entanglement vanishes in finite time (sudden death) [49]. This is in contrast to the loss of quantum coherence, which is usually gradual $[30,31,49]$.

The logarithmic negativity, shown in Fig. 6(a) decays very fast for model A as compared to model B in Fig. 6(b). In other words, the entanglement can be sustained much longer when two subsystems are coupled to a common bath than to 

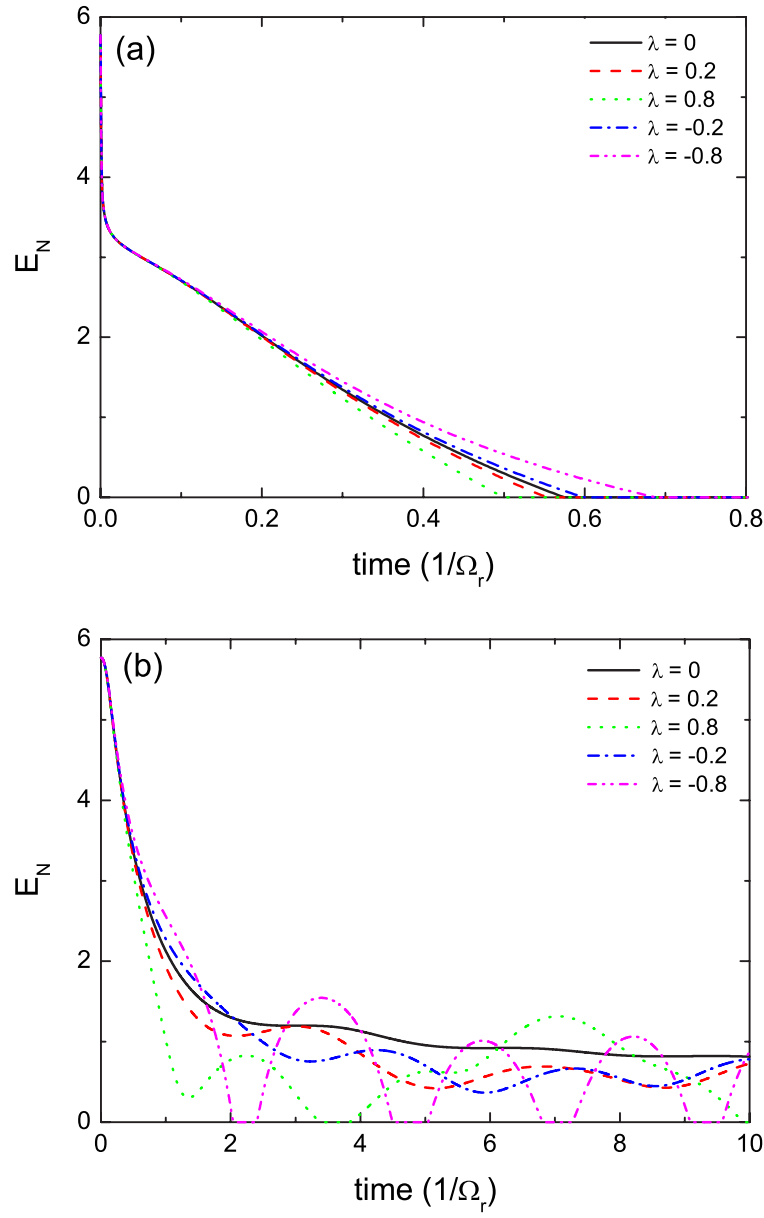

FIG. 6. (Color online) Time evolutions of the logarithmic negativity of the two subsystems coupled more strongly to the environments $\left(\gamma_{0}=6 \times 10^{-2} \Omega_{r}\right)$ for an initial two-mode squeezed vacuum state with a squeezing parameter $r=2$. (a) is for model A and (b) for model $\mathrm{B}$. The solid line is for $\lambda=0$, dashed for $\lambda=0.2$, dotted for $\lambda=0.8$, dash-dotted for $\lambda=-0.2$, and dash-dot-dotted for $\lambda=-0.8$.

individually independent baths. This conclusion is consistent with the result found in other continuous variable models $[25,28]$ or discrete qubit models [50,51]. In our models, this can be understood by noting that the Hamiltonian of the total system can be written in terms of new dynamical variables, the sum and difference of the two oscillators' positions and momenta $\left(x+y, p_{x}+p_{y}, x-y, p_{x}-p_{y}\right)$. For model B coupled to a common environment, only the mode of the sum of the two positions interacts with the environment, and the mode of the difference of the two positions undergoes a free evolution. As a result, only the modes of the sum of the positions and momenta are affected by the environment [see Eq. (23)]. But for model $\mathrm{A}$, these modes all interact with the environments [see Eq. (10)] and thus are all influenced by the environments.

In Fig. 7(a), we find that the logarithmic negativity for model A is barely generated with an initially separable state ( $r=0$ case). On the other hand, we find in Fig. 7(b) that even with no interaction between the two subsystems, because they are coupled to a common bath (model B), the entanglement can be generated from an initially separable state $(r$
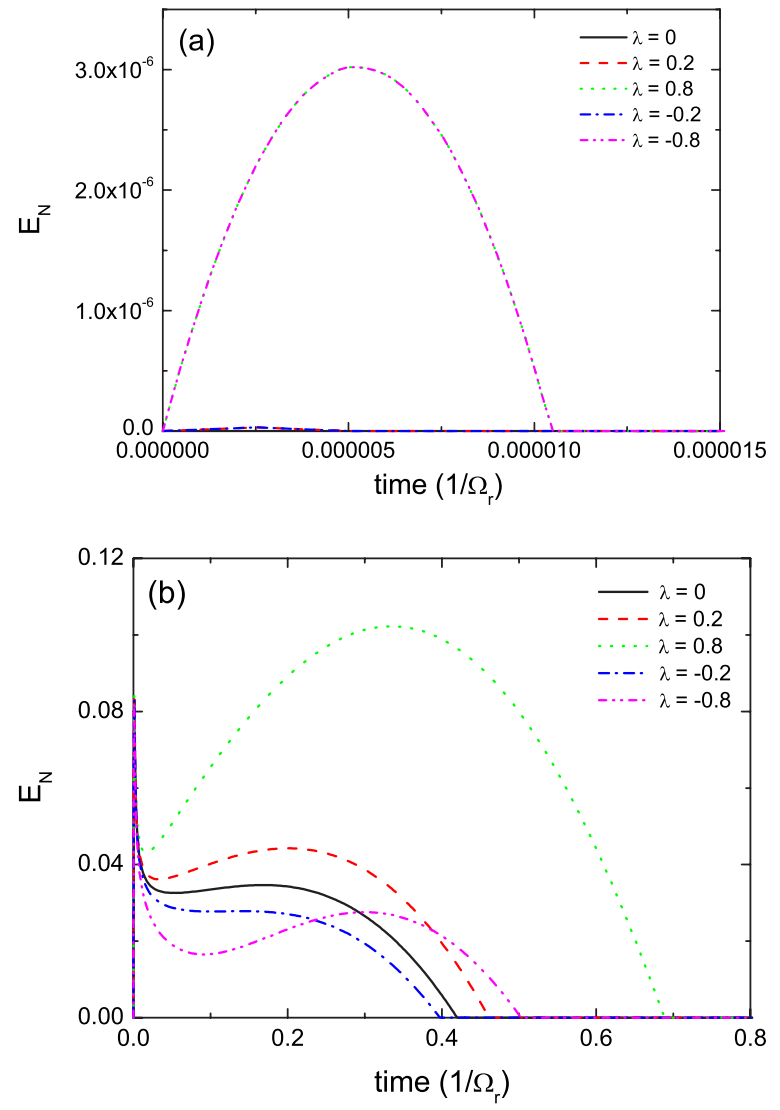

FIG. 7. (Color online) Time evolutions of the logarithmic negativity for an initial squeezing parameter $r=0$. Other conditions and plot caption are the same as in Fig. 6.

$=0$ case $)[25,28,50,51]$. But the generated entanglement lasts only for a short time and then disappears. In most situations the entanglement is created for a very short time after the interaction with the environment is turned on. The entanglement may persist for long times or disappear quickly, depending on the system-environment coupling and the properties of the environment $[25,28,50,51]$.

\section{Condition for entanglement survival}

A condition derived in Ref. [25] stated that, if the twomode squeezed state of the electromagnetic field embedded in a thermal environment is initially sufficiently squeezed, it will always remain entangled independently of the strength of the interaction to the environment. Each of the two electromagnetic modes has the Hilbert space equivalent to the Hilbert space of a harmonic oscillator. As a result, the model studied in Ref. [25] is the same as our model B when $\lambda=0$. However, the conclusion in Ref. [25] was reached using the RWA and Markovian master equation. Here we investigate whether the condition presented in Ref. [25] is still valid or needs some modification in the non-Markovian case.

In Ref. [25], the Simon criterion [47] for continuous variables system was used to verify whether the quantum state of the system is entangled or separable. It was found that if the initial state is sufficiently squeezed, i.e., the squeezed param- 

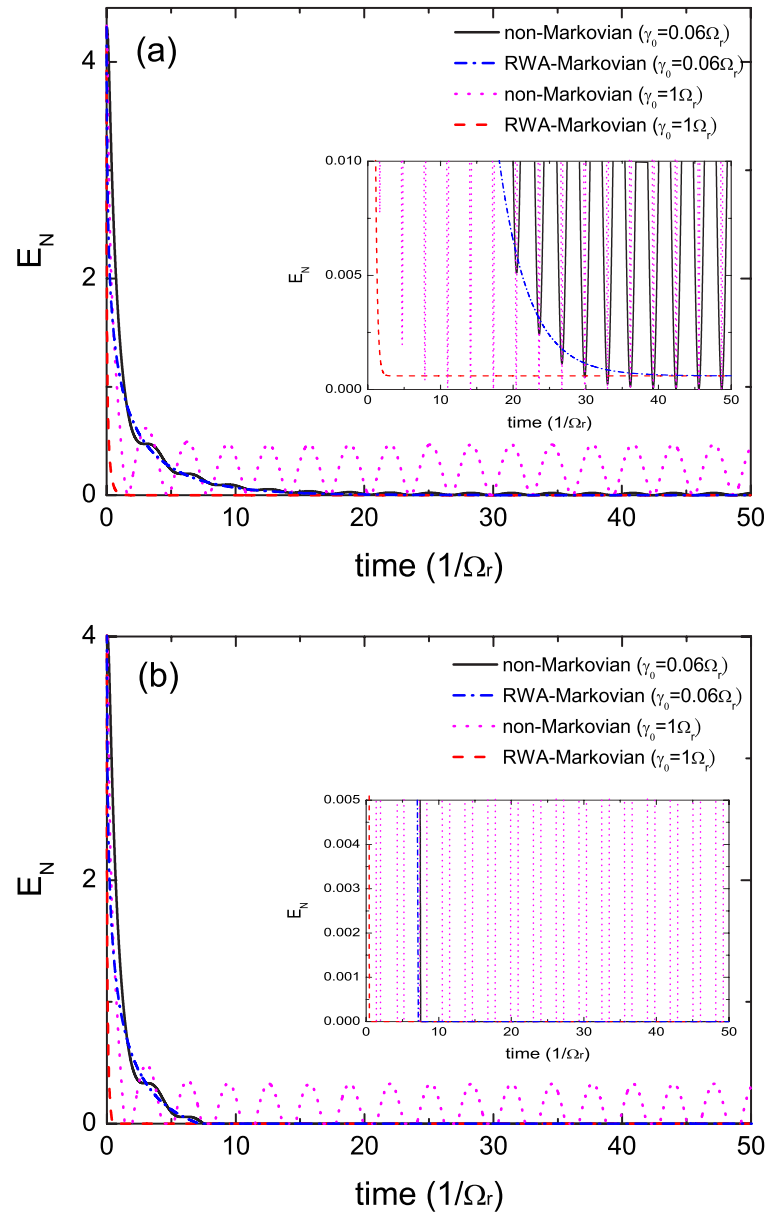

FIG. 8. (Color online) Time evolutions of the logarithmic negativity for different initial squeezed parameters $r=$ (a) 1.489 and (b) 1.4. Two different values of $\gamma_{0}\left(\gamma_{0}=0.06 \Omega_{r}\right.$ and $\left.\gamma_{0}=1 \Omega_{r}\right)$ are used in each plot, where $\gamma_{0}$ is related to the system-environment coupling strength. The insets illustrate the same plots but with much smaller values of vertical axis $E_{N}$.

eter of the initial two-mode squeezed vacuum state satisfies [25]

$$
|r| \geqslant \frac{1}{2} \ln (2 \bar{N}+1)
$$

where

$$
\bar{N}=\frac{1}{e^{\hbar \Omega_{r} / k_{B} T}-1}
$$

is the mean thermal photon number, it will remain entangled forever in spite of the interaction between the system and the external environment. Otherwise, the state will disentangle (become separable) after time [25]

$$
t=\frac{1}{2 \gamma} \ln \left(\frac{2 \bar{N}+1-e^{-2|r|}}{2 \bar{N}+1-e^{2|r|}}\right),
$$

where $\gamma \approx 2 \lim _{t \rightarrow \infty} \gamma(t) \approx 2 \gamma_{0}$. From Eqs. (42) and (43), at a temperature of $k_{B} T=10 \hbar \Omega_{r}$, the corresponding critical squeezed parameter is $\left|r_{c}\right|=\frac{1}{2} \ln (2 \bar{N}+1)=1.498$. We choose the squeezing parameters to be at and slightly smaller than this critical squeezing value, and vary the systemenvironment interaction strengths to check whether the condition for the inequality (42) is still valid.

Form Fig. 8(a) and its inset the logarithmic negativity does not vanish and appears cyclically at long times for $r$ $=r_{c}=1.498$ regardless of their system-environment interaction strength. So the statement about the inequality (42) in Ref. [25] seems valid for both non-Markovian and RWA Markovian cases. On the other hand, if the squeezed parameter $r=1.4<r_{c}$, i.e., smaller than the critical squeezed parameter, Eq. (44) predicts that the two-mode state will disentangled (or become separable) after time $t=7.168 / \Omega_{r}$ for $\gamma_{0}=0.06 \Omega_{r}$ and $t=0.43 / \Omega_{r}$ for $\gamma_{0}=1 \Omega_{r}$. This is indeed the case for the RWA-Markovian approximation results shown in Fig. 8(b) for our model B with $\lambda=0$. However, this is not true for the non-Markovian case. We find that entanglement disappears except for the non-Markovian case with a larger coupling strength $\gamma_{0}$, in which the entanglement dies out first and then is generated cyclically by the interaction with the common bath [see Fig. 8(b) and its inset]. In other words, the non-Markovian dynamics predicts that the entanglement will persist for a longer time. This is consistent with the result in Ref. [52]. So in the case of non-Markovian dynamics, the inequality (42) and Eq. (44) are no longer true, and the condition not only depends on the mean thermal photon number but also on the interaction strength between the system and the environment.

\section{CONCLUSION}

We have investigated the non-Markovian entanglement dynamics of two oscillator subsystems which are coupled to a common environment or are coupled, respectively, to their own independent environments. We have presented and discussed the influence of the environments on the entanglement dynamics by varying initial states (different squeezing parameters), oscillator-oscillator interactions, and oscillatorenvironment interactions. We have found that the dynamics of entanglement oscillates for two interacting subsystems isolated from the external environment. The attractive interaction seems always to be better than the repulsive interaction as far as the maximum value of entanglement that can be reached at a later time is concerned. When the coupling between the environments and the two subsystems is turned on and increased progressively, these periodic behaviors die down gradually and disappear eventually. When the interaction strength to the environments is increased further, the entanglement vanishes at finite times (sudden death). This is in contrast to the loss of quantum coherence, which is usually gradual. It is also been found that the entanglement can be sustained much longer when the two subsystems are coupled to a common bath than to individually independent baths. This conclusion is consistent with the results found in other models [25]. In summary, the dynamics of the quantum entanglement is sensitive to the initial states, the oscillator 
-oscillator interaction, the oscillator-environment interaction, and the coupling to a common bath or to different, independent baths.

Finally, we have checked the condition for entanglement to exist at long times for two noninteracting subsystems coupled to a common bath (model B with $\lambda=0$ ). In contrast to the condition, which depends only on the mean thermal phonon number, obtained in Ref. [25] using the RWA Markovian master equation, our non-Markovian analysis indicates that the condition also depends on the systemenvironment interaction.

\section{ACKNOWLEDGMENTS}

We would like to acknowledge support from the National Science Council, Taiwan, under Grants No. NSC95-2112-M002-018 and No. NSC95-2112-M-002-054. We also acknowledge support from the focus group program of the National Center for Theoretical Sciences, Taiwan. H.S.G. acknowledges support from the National Taiwan University under Grant No. 95R0034-02, thanks C. H. Chou and B. L. $\mathrm{Hu}$ for useful discussions, and is grateful to the National Center for High-Performance Computing, Taiwan, for computer time and facilities.
[1] S. L. Braunstein and P. van Loock, Rev. Mod. Phys. 77, 513 (2005).

[2] A. Furusawa, J. L. Sorensen, S. L. Braunstein, C. A. Fuchs, H. J. Kimble, and E. S. Polzik, Science 282, 706 (1998).

[3] T. C. Zhang, K. W. Goh, C. W. Chou, P. Lodahl, and H. J. Kimble, Phys. Rev. A 67, 033802 (2003).

[4] W. P. Bowen, N. Treps, B. C. Buchler, R. Schnabel, T. C. Ralph, Hans-A. Bachor, T. Symul, and P. Koy, Phys. Rev. A 67, 032302 (2003).

[5] H. P. Yuen and A. Kim, Phys. Lett. A 241, 135 (1998); 246, 560(E) (1998).

[6] F. Grosshans, G. Van Assche, J. Wenger, R. Brouri, N. J. Cerf, and P. Grangier, Nature (London) 421, 238 (2003).

[7] M. Roukes, Phys. World 14 (Feb.), 25 (2001); H. G. Craighhead, Science 290, 1532 (2000).

[8] M. Blencowe, Phys. Rep. 395, 159 (2004).

[9] R. G. Knobel and A. N. Cleland, Nature (London) 424, 291 (2003).

[10] M. D. LaHaye, O. Buu, B. Camarota, and K. C. Schwab, Science 304, 74 (2004).

[11] A. Naik, O. Buu, M. D. LaHaye, A. D. Armour, A. A. Clerk, M. P. Blencowe, and K. C. Schwab, Nature (London) 443, 193 (2006).

[12] A. D. Armour, M. P. Blencowe, and K. C. Schwab, Phys. Rev. Lett. 88, 148301 (2002).

[13] W. Marshall, C. Simon, R. Penrose, and D. Bouwmeester, Phys. Rev. Lett. 91, 130401 (2003).

[14] S. Mancini, V. Giovannetti, D. Vitali, and P. Tombesi, Phys. Rev. Lett. 88, 120401 (2002).

[15] S. Pirandola, D. Vitali, P. Tombesi, and S. Lloyd, Phys. Rev. Lett. 97, 150403 (2006).

[16] F. Xue, Y.-X. Liu, C. P. Sun, and F. Nori, e-print arXiv:quant$\mathrm{ph} / 0701209$.

[17] K. Audenaert, J. Eisert, M. B. Plenio, and R. F. Werner, Phys. Rev. A 66, 042327 (2002).

[18] M. B. Plenio, J. Hartley, and J. Eisert, New J. Phys. 6, 36 (2004).

[19] J. Eisert, M. B. Plenio, S. Bose, and J. Hartley, Phys. Rev. Lett. 93, 190402 (2004).

[20] L.-M. Duan and G.-C. Guo, Quantum Semiclassic. Opt. 9, 953 (1997).

[21] T. Hiroshima, Phys. Rev. A 63, 022305 (2001).

[22] S. Scheel and D.-G. Welsch, Phys. Rev. A 64, 063811 (2001).
[23] D. Wilson, J. Lee, and M. S. Kim, J. Opt. Soc. Am. B 50, 1809 (2003).

[24] S. Olivares, M. G. A. Paris, and A. R. Rossi, Phys. Lett. A 319, 32 (2003).

[25] J. S. Prauzner-Bechcicki, J. Phys. A 37, L173 (2004).

[26] A. Serafini, F. Illuminati, M. G. A. Paris, and S. De Siena, Phys. Rev. A 69, 022318 (2004).

[27] A. Serafini, M. G. A. Paris, F. Illuminati, and S. De Siena, J. Opt. Soc. Am. B 7, R19 (2005).

[28] F. Benatti and R. Floreanini, J. Phys. A 39, 2689 (2006).

[29] M. Ban, J. Phys. A 39, 1927 (2006).

[30] P. J. Dodd and J. J. Halliwell, Phys. Rev. A 69, 052105 (2004).

[31] P. J. Dodd, Phys. Rev. A 69, 052106 (2004).

[32] C. W. Gardiner and P. Zoller, Quantum Noise, 2nd ed. (Springer, Berlin, 2000).

[33] X. M. H. Huang, C. A. Zorman, M. Mehregany, and M. L. Roukes, Nature (London) 421, 496 (2003).

[34] W. K. Hensinger, D. W. Utami, H.-S. Goan, K. Schwab, C. Monroe, and G. J. Milburn, Phys. Rev. A 72, 041405(R) (2005).

[35] J. P. Paz and W. H. Zurek in Coherent Matter Waves, Proceedings of the Les Houches Summer School, Session LXXII, edited by R. Kaiser, C. Westbrook, and F. David (SpringerVerlag, Berlin, 2001 ); e-print arXiv:quant-ph/0010011.

[36] A. J. Leggett et al., Rev. Mod. Phys. 59, 1 (1987).

[37] B. L. Hu, J. P. Paz, and Y. Zhang, Phys. Rev. D 45, 2843 (1992).

[38] C. H. Chou and B. L. Hu (private communication).

[39] L. H. Ryder, Quantum Field Theory, 2nd ed. (Cambridge University Press, Cambridge, U.K., 1996).

[40] F. Mandl and G. Shaw, Quantum Field Theory, rev. ed. (John Wiley \& Sons, Chichester, 1993).

[41] A. O. Caldeira and A. J. Legget, Ann. Phys. (N.Y.) 149, 374 (1983); 153, 445(E) (1983).

[42] U. Weiss, Quantum Dissipative Systems, 2nd ed. (World Scientific, Singapore, 1999).

[43] G. Adesso, A. Serafini, and F. Illuminati, Phys. Rev. A 70, 022318 (2004).

[44] G. Vidal and R. F. Werner, Phys. Rev. A 65, 032314 (2002).

[45] M. B. Plenio, Phys. Rev. Lett. 95, 090503 (2005).

[46] J. Laurat, G. Keller, J. A. Oliveira-Huguenin, C. Fabre, T. Coudreau, A. Serafini, G. Adesso, and F. Illuminati, J. Opt. B: Quantum Semiclassical Opt. 7, S577 (2005). 
[47] R. Simon, Phys. Rev. Lett. 84, 2726 (2000).

[48] L.-M. Duan, G. Giedke, J. I. Cirac, and P. Zoller, Phys. Rev. Lett. 84, 2722 (2000).

[49] T. Yu and J. H. Eberly, Phys. Rev. Lett. 93, 140404 (2004).

[50] D. Braun, Phys. Rev. Lett. 89, 277901 (2002).
[51] F. Benatti, R. Floreanini, and M. Piani, Phys. Rev. Lett. 91, 070402 (2003).

[52] S. Maniscalco, S. Olivares, and M. G. A. Paris,Phys. Rev. A 75, 062119 (2007). 